This item was submitted to Loughborough's Research Repository by the author.

Items in Figshare are protected by copyright, with all rights reserved, unless otherwise indicated.

\title{
Haematoma detection using EIT in a sheep model
}

PLEASE CITE THE PUBLISHED VERSION

http://dx.doi.org/10.2316/P.2013.791-112

PUBLISHER

(C) ACTA Press

VERSION

AM (Accepted Manuscript)

LICENCE

CC BY-NC-ND 4.0

REPOSITORY RECORD

Ayati, S. Bentolhoda, Kaddour Bouazza-Marouf, David Kerr, and Michael D. O'Toole. 2019. "Haematoma Detection Using EIT in a Sheep Model". figshare. https://hdl.handle.net/2134/12094. 
This item was submitted to Loughborough's Institutional Repository (https://dspace.lboro.ac.uk/) by the author and is made available under the following Creative Commons Licence conditions.

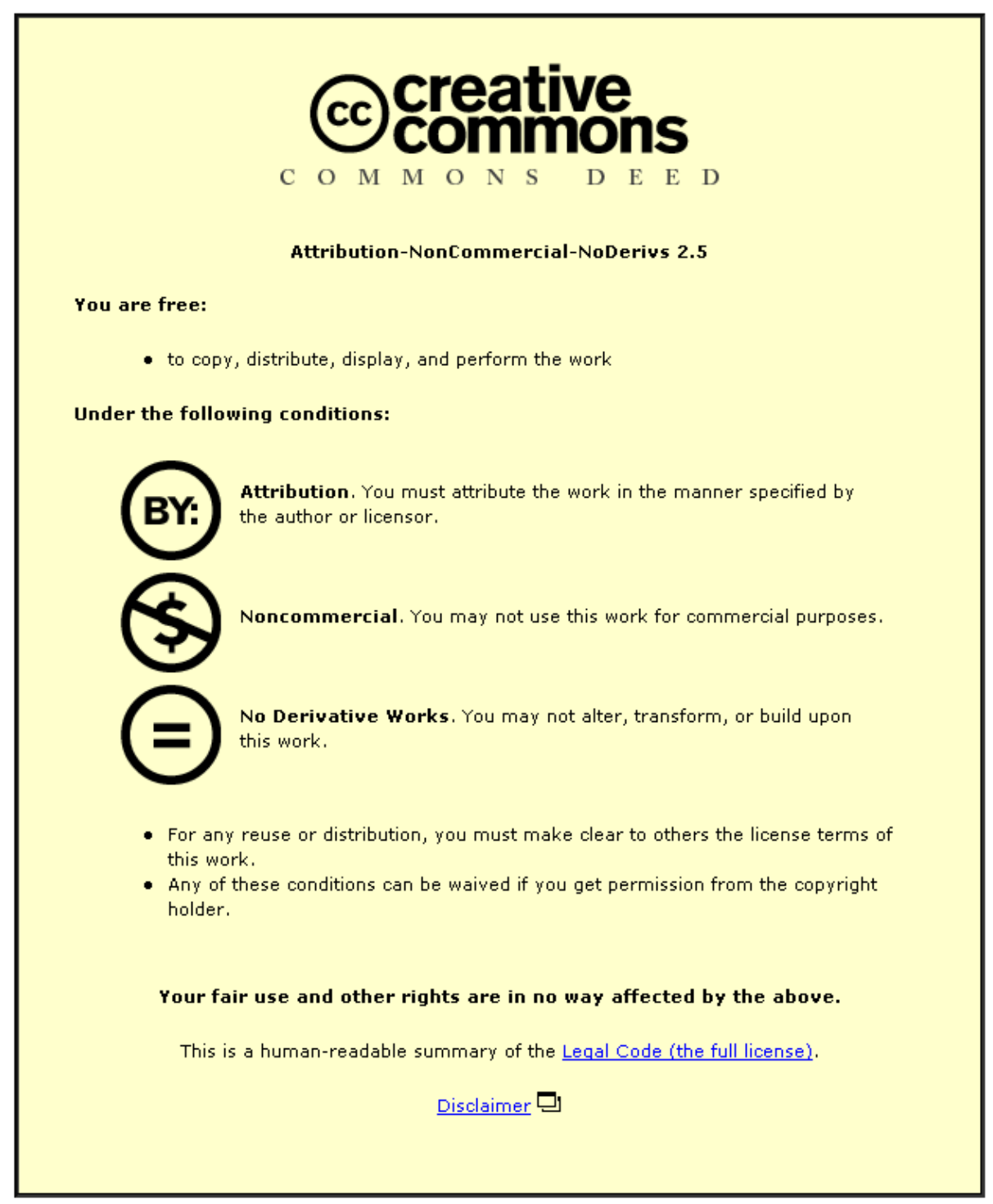

For the full text of this licence, please go to: http://creativecommons.org/licenses/by-nc-nd/2.5/ 


\title{
HAEMATOMA DETECTION USING EIT IN A SHEEP MODEL
}

\author{
S. Bentolhoda Ayati ${ }^{\mathrm{a}}$, Kaddour Bouazza-Marouf ${ }^{\mathrm{b}}$, David Kerr ${ }^{\mathrm{c}}$, Michael O’Toole ${ }^{\mathrm{d}}$ \\ a, b, c Wolfson School of Mechanical and Manufacturing Engineering, Loughborough University, Loughborough, LE11 3TU, \\ UK \\ ${ }^{\mathrm{d}}$ School of Computer Science, University of Manchester, Oxford Rd, Manchester, M13 9PL, UK \\ a s.b.ayati@lboro.ac.uk, ${ }^{\mathrm{b}}$ K.Bouazza-marouf@lboro.ac.uk, D.Kerr@lboro.ac.uk, ${ }^{\mathrm{d}}$ Michael.OToole@cs.man.ac.uk
}

\begin{abstract}
Performance evaluation of a portable digital electrical impedance tomography system to detect haematomas using a sheep model is presented. Two different experiments have been performed using 8-electrode full array configuration. Artificial haematomas were introduced in the first experiment by injecting blood-like conductivity solution via the brainstem, and in the second by placing blood-like conductivity gel at a certain position on top of the parietal lobes of the brain on the left and right sides. For the first experiment, the Electrical Impedance Tomography (EIT) images were reconstructed sequentially for different injection volumes and the quantity index (QI) was calculated as a function of the injected solution volume. The results show a linear relationship of QI to the injected volume. For the second experiment, the images were successfully reconstructed and haematoma was clearly detected and localised using our developed system. The promising results of sheep experiments prove that our developed EIT system is able to detect and quantify small haematomas in head.
\end{abstract}

\section{KEY WORDS}

Electrical Impedance Tomography, Haematoma, Sheep Model, Assistive Medical Technology, Medical Image Processing

\section{Introduction}

Intracranial haematomas are pockets of blood that buildup within the cranial vault as a result of haemorrhage from a head injury. The haemorrhage is an active bleeding and the haematoma's excess blood is not reabsorbed into the body system and may also increase in size and become a serious condition. These pockets expand and increase the intracranial pressure on the brain, causing impairment of the ability of the brain to function and worsening the neurological condition of the patient. If left unchecked, the growing haematoma will cause severe and even permanent damage to the delicate tissue of the brain, the morbidity, and eventual death of the patient. The development of the haematoma from benign to symptomatic can be sudden, and a patient can change from lucid to a state of rapid neurological deterioration over a very short period of time [1].
Acute imaging of the head is the first action when a patient arrives in the emergency department on suspicion of head injury to identify the necessity of urgent (treatments) surgical intervention or stabilization to prevent further injury. The current appropriate imaging technique used in the acute phase is CT scanning. However CT scanning or MRI in the UK are not always available for 24 hours a day, and in cases of multiple traumas, it may not be possible to scan the patient until they have been adequately stabilised. The transporting requirement of ill patients and equipment compatibility restrict using this method for the acute care. These imaging techniques are not portable and suitable for continuous monitoring and involve large personnel or equipment cost [2].

There is a direct and urgent clinical need for a robust, easy to use and low-cost system which can accurately detect the presence of a haematoma in high risk patients. A device of this nature can reduce treatment delays, save on costs and waste, and most significantly, positively impact patient outcomes. It also provides a primary and possibly sole method of haematoma detection when CT scanning or MRI is unavailable or impossible. Treatment delays are mitigated by giving better and earlier information on haematomas at the triage stage. The time from injury to treatment is a key factor in patient recovery, and earlier diagnosis leads to earlier treatment.

Electrical impedance tomography (EIT) is a noninvasive, non-radiating, non-ionizing and inexpensive technique. EIT has the capability of long term monitoring and can be used to approximately localize the bleeding site. EIT is a medical imaging technique which reconstructs cross-sectional images of the electrical property distribution of an object within a body based on voltage measurements on its boundary. Data acquisition is performed through an array of electrodes which are attached to the surface of the body. EIT has been extensively researched in various clinical applications due to its potential to distinguish between healthy and abnormal components of an organ [3].

EIT is a relatively new medical imaging modality and present significant possibilities for medical application, including head and abdominal imaging [3]. In terms of bleeding, a study has presented that EIT can detect approximately $30 \mathrm{ml}$ of blood in the peritoneum of neonatal piglets [4]. The aim of this paper is to evaluate the sensitivity of a digital EIT system constructed in our 


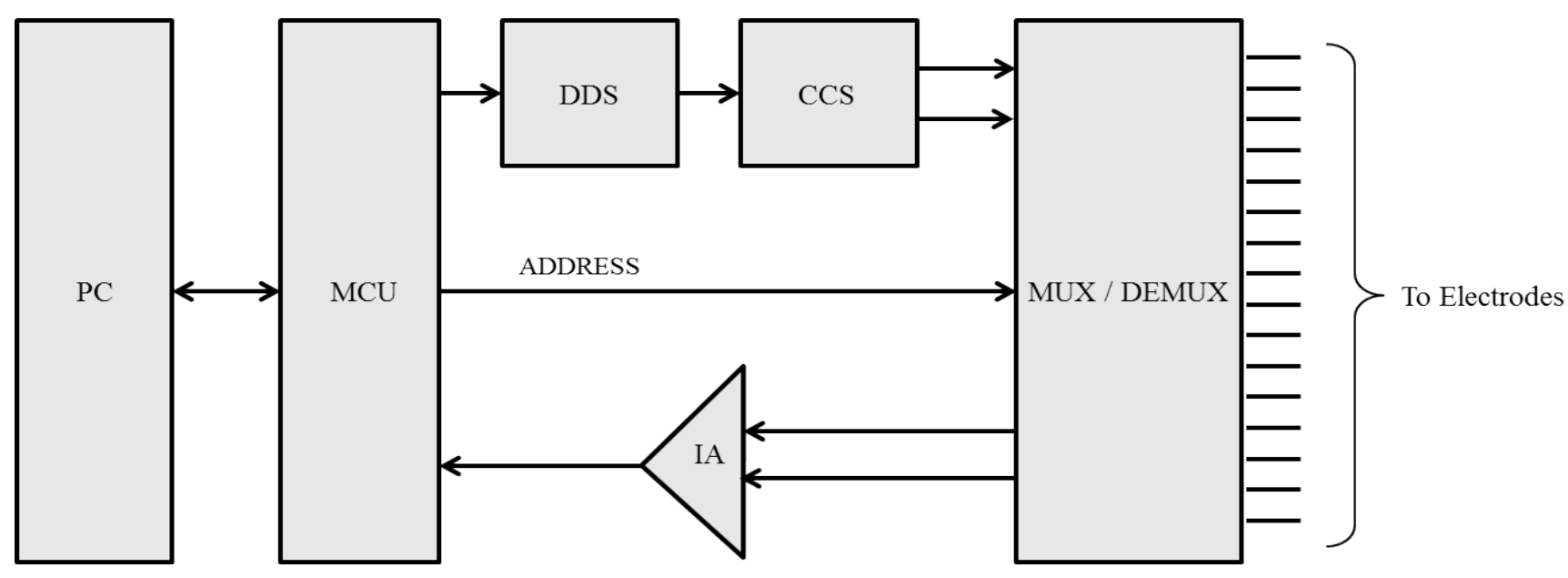

Figure 1. EIT system architecture

laboratory to detect and localise simulated haematomas, 1 $\mathrm{ml}$ to $10 \mathrm{ml}$, in a sheep's head and to study the relationship between the injected simulated blood and the EIT images.

\section{Materials and Methods}

\subsection{System Specification and Methods}

The structure of the designed EIT system based on a microcontroller is presented in Figure 1. The system is controlled by a microcontroller connected to a PC through a serial port (RS232). A graphical user interface was developed using Visual Basic (VB). The Constant Current Source, CCS generates a constant sinusoidal current fed by a sinusoidal signal of the same frequency generated by the Direct Digital Synthesizer, DDS.

The current was injected through one pair of adjacent electrodes and the corresponding boundary potentials were measured over pairs of the remainder of the neighbouring electrodes using a multiplexer. The input pair of electrodes was switched over all adjacent pair electrodes and the measurement procedure was repeated for all possible adjacent pairs. The measurements were amplified using an Instrumentation Amplifier (IA) to produce a complete voltage data set. The performance of this system was previously evaluated using phantom experiments [5].

This multi-frequency EIT system has 16 channels and operates in the frequency range of $25 \mathrm{kHz}$ to $100 \mathrm{kHz}$ with the temporal resolution of 100 frames per second. For this experiment, a constant current of $1 \mathrm{~mA}$ at a single frequency of $50 \mathrm{kHz}$ and 8 electrodes were chosen.

\subsection{Sheep Preparation}

Two fresh sheep heads were obtained from a local butcher and skinned. The locations of the 8 electrodes of the full array configuration were marked on the skull with the equal distance between electrodes. Eight $10 \mathrm{~mm} \mathrm{Ag/AgCl}$ disk electrodes (Unimed Electrode Supplies Ltd) were fastened to the skull using conductive paste (Unimed Electrode Supplies Ltd).

A saline solution with the same conductivity as blood $(0.67 \mathrm{~S} / \mathrm{m})$ was made with the concentration of $0.33 \%$ [weight/volume] of sodium chloride in water. In the first sheep's head the blood-equivalent saline was injected near the ventricles via the brainstem. The injections were made in increments of $0.5 \mathrm{ml}$ to $9 \mathrm{ml}$ using a long syringe. Blood has higher conductivity than brain tissue $(0.17 \mathrm{~S} / \mathrm{m})$ thus increases in blood volume should result in increase of conductivity increment in the reconstructed images [6].

In the above experiment, blood was spread out inside the skull without any limitation because of the absence of CSF and brain pressure. In order to simulate a more realistic haematoma in an accurate location, the saline solution was transformed to gel. The saline solution was stirred using a magnetic stir bar at a temperature of $70^{\circ} \mathrm{C}$ while agar powder (Fisher Scientific) was added to achieve the desired gel concentration (1.7\% by weight). Then the solution was poured into a tube with the diameter of $1 \mathrm{~cm}$ and allowed to cool at room temperature. The gel sample was removed from the tube and cut to lengths of $3 \mathrm{~mm}$ to use as pockets of blood. The conductivity of the gel sample was measured and found to be the same as the conductivity of the saline solution. An AC voltage was applied across the gel at a frequency of 50 $\mathrm{kHz}$ at room temperature using a waveform function generator connected in series with a digital multimeter to measure the AC current and AC voltage across the gel. 


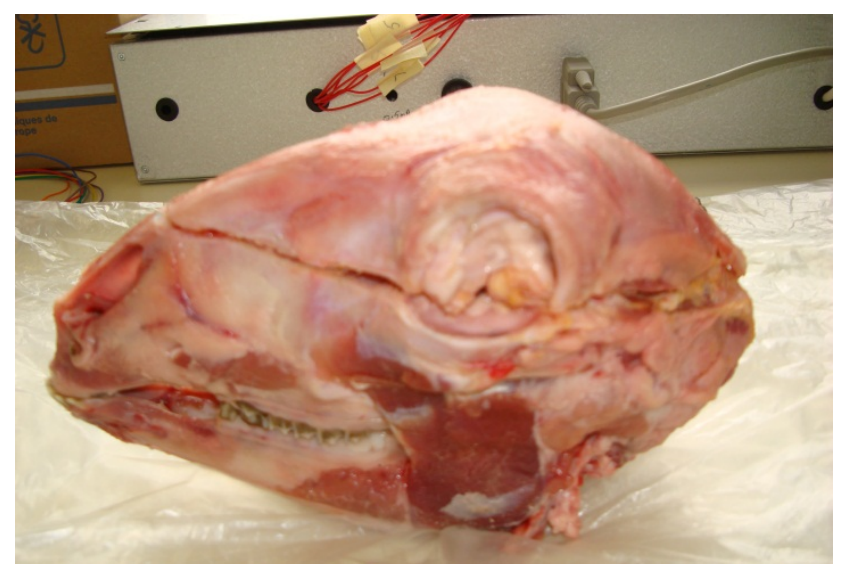

a)

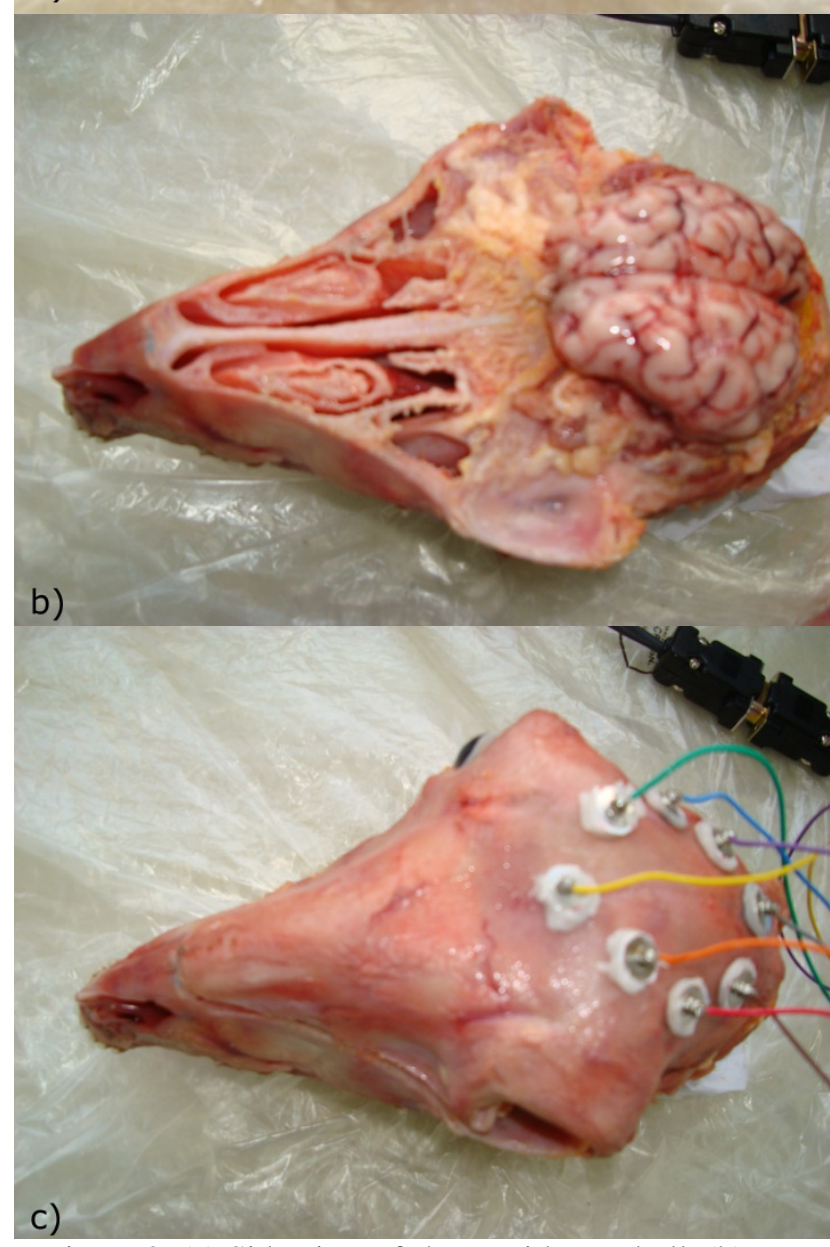

Figure 2. (a) Side view of sheep with cut skull. (b) top view with complete brain out. (c) top view with full array electrodes attached

For the second sheep's head, the skull was cut approximately in half as shown in Figure 2 using a bone saw. The top half of the skull was carefully removed and the brain was exposed. A gel sample was placed on top of the parietal lobes at approximately the middle of the posterior quarter of the brain on the left and right sides. The top half of the skull was replaced and the measurement was performed.

\subsection{Reconstruction and Processing}

EIT is composed of forward problem and inverse problem. The forward problem is to predict the measured boundary electric potentials from the known applied current density and conductivity distribution. The aim of the inverse problem which is also known as image reconstruction is to estimate the conductivity distribution from a known set of boundary voltage measurements.

For the forward problem the complete electrode model was used and defined by the following equations:

$$
\begin{array}{ll}
\nabla .(\sigma \nabla \mathrm{u})=0 & \text { on } \Omega \\
\mathrm{u}+\mathrm{z}_{\mathrm{l}} \sigma \frac{\partial \mathrm{u}}{\partial \mathrm{n}}=\mathrm{V}_{\mathrm{l}} & \text { on } \varepsilon_{1}, \mathrm{l}=1,2, \ldots, \mathrm{L} \\
\int_{\varepsilon_{1}} \sigma \frac{\partial \mathrm{u}}{\partial \mathrm{n}} \mathrm{ds}=\mathrm{I}_{\mathrm{l}} & \text { on } \varepsilon_{1}, \mathrm{l}=1,2, \ldots, \mathrm{L} \\
\sigma \frac{\partial \mathrm{u}}{\partial \mathrm{n}}=0 & \text { on } \partial \Omega \backslash\left\{\varepsilon_{1} \cup \varepsilon_{2} \cup \ldots, \varepsilon_{\mathrm{L}}\right\}
\end{array}
$$

To provide the forward solution, the Finite Element Method (FEM) was used in Comsol (Comsol Multiphysics).

The images were reconstructed using the sensitivity method. The sensitivity matrix was calculated from forward solutions of a two-dimensional disk model. The model was discretized to 3032 second-order triangular finite elements. The conductivity distribution of the brain tissue is generally not known a priori and therefore $\mathrm{S}$ was calculated assuming a homogenous conductivity distribution. To determine the conductivity changes, the sensitivity matrix was inverted:

$$
\Delta \sigma \cong S^{\dagger} \Delta V
$$

where $S^{\dagger}$ is the Moore-Penrose pseudo inverse of $S$. Since the EIT inverse problem is severely ill-posed and a small amount of noise on boundary measurements, $\Delta V$, can cause a large oscillation for the solution, $\Delta \sigma$, a regularization technique was used to reduce this effect by improving the conditioning of $S$. The truncated Singular Value Decomposition (TSVD) method which has previously been identified as a suitable regularization method [7] was used to regularize the inversion of the sensitivity matrix. The truncation point $k$, needs to be chosen carefully, less than or equal to the rank of the matrix, as it would otherwise produce inaccurate images. The truncation point was chosen as 16 on inspection of the L-curve of experimental data [8].

The quantity index (QI) is defined as an EIT image parameter that correlates with the anomaly volume. The quantity index (QI) is the integral of conductivity change multiplied by the area of the element over the image area:

$$
Q I=\sum_{i=1}^{n e} \Delta \sigma_{i} a_{i}
$$


where, for an element (or pixel) $i, \Delta \sigma_{i}$ is the conductivity change reconstructed in the $i$-th element, $a_{i}$ is the area of each element and $n e$ is the total number of elements.

The position of the anomaly ( $\mathrm{x}, \mathrm{y})$ can be estimated from reconstructed images using:

$$
\begin{aligned}
& \mathrm{x}=\frac{\sum_{i=1}^{n e} \Delta \sigma_{i} a_{i} x_{i}}{\sum_{i=1}^{n e} \Delta \sigma_{i} a_{i}} \\
& \mathrm{y}=\frac{\sum_{i=1}^{n e} \Delta \sigma_{i} a_{i} y_{i}}{\sum_{i=1}^{n e} \Delta \sigma_{i} a_{i}}
\end{aligned}
$$

The expression for anomaly location $(\mathrm{x}, \mathrm{y})$ is effectively the centre of the reconstructed anomaly. The values $x_{i}$ and $y_{i}$ are the coordinates of the centre of each element. Diametric conductivity plot (DCP) is the conductivity difference profile of the model based on the diameter passing through the reconstructed object center. It is plotted in terms of the arc-length of the diameter.

\section{Results}

In the first sheep's head, where the blood-like solution was injected into the ventricles, the location of the solution was not accurately known. Therefore, the EIT images were reconstructed sequentially and the quantity index was calculated as a function of the injected solution volume as shown in Figure 3. The plot shows the linear relationship between QI and injected volume. The calculated QI increased as more solution is injected.

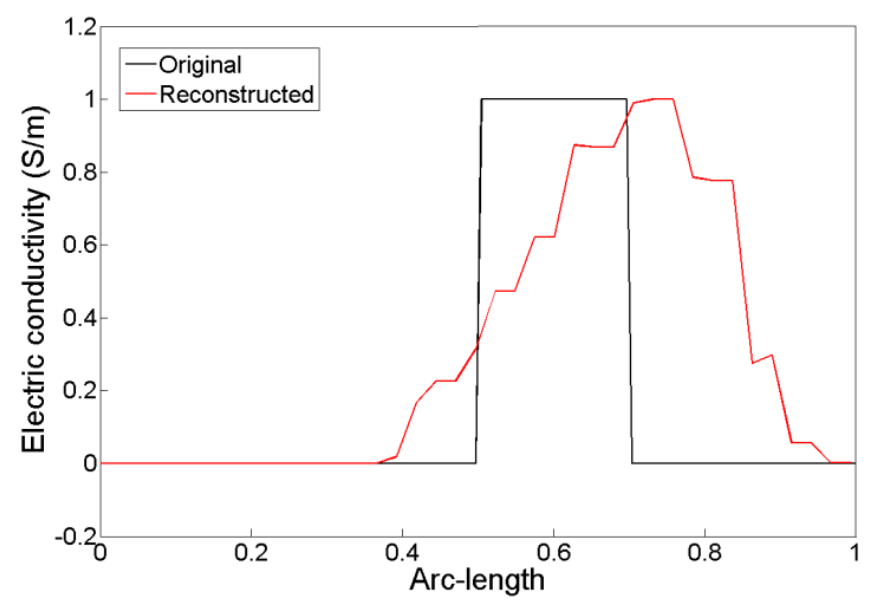

Figure 4. DCP and reconstructed image of the haematoma on top of the left parietal lobe
In the second sheep's head, a simulated haematoma was placed at a certain position. DCP and the reconstructed image of the brain with such a haematoma placed on top of the left parietal lobe are shown in Figure 4. It can be seen that the image is successfully reconstructed and the haematoma is clearly detected. In the image, the red region (which represents a conductivity increase of approximately $1 \mathrm{~S} / \mathrm{m}$ ) appeared in the image in accordance with the position of the haematoma. It is observed that the reconstructed DCP almost follows the DCP of the original object. The center of the reconstructed anomaly on the left side was calculated as $(-0.5240 \mathrm{R}$, $0.4584 \mathrm{R})$ where $\mathrm{R}$ is the radius of the imaged brain.

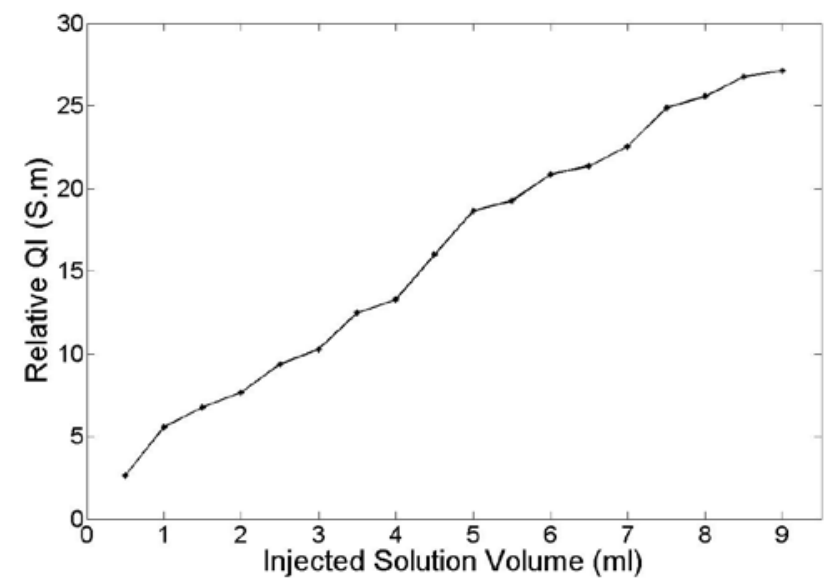

Figure 3. Quantity index as a function of injected saline solution volume

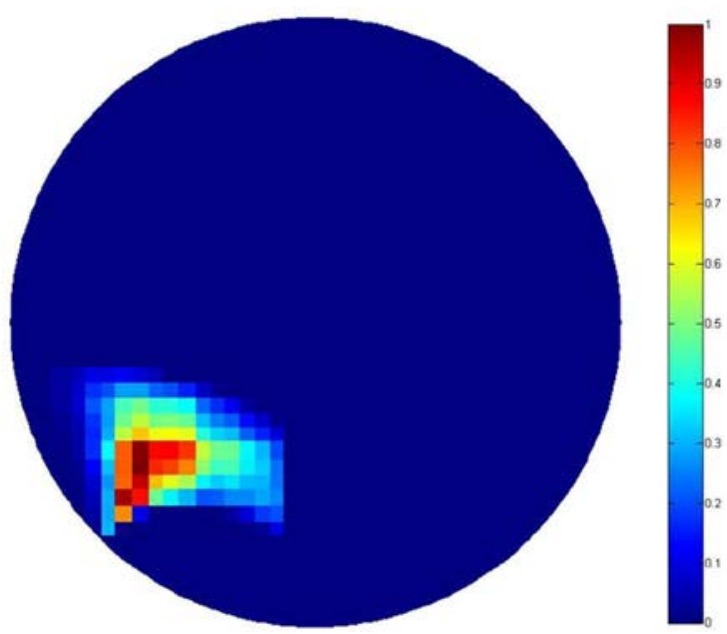



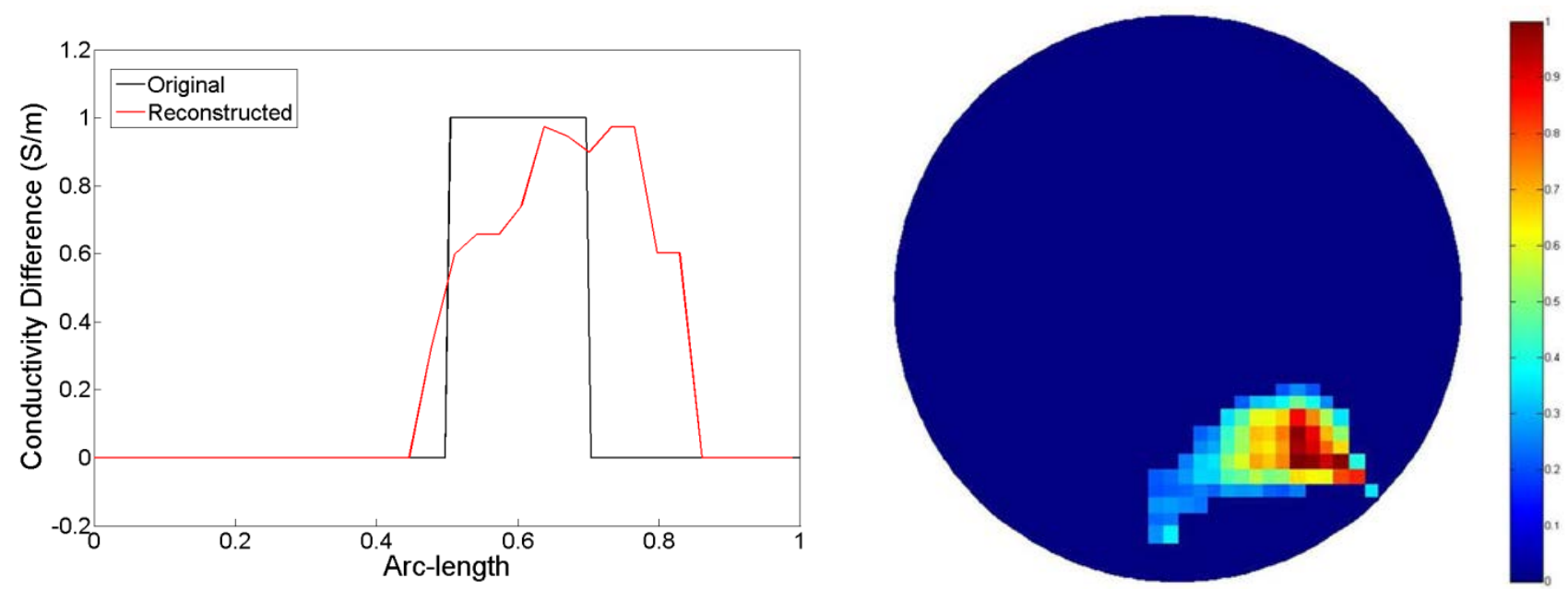

Figure 5. DCP and reconstructed image of the haematoma on top of the right parietal lobe

DCP and the reconstructed image of the brain with a haematoma placed on top of the right parietal lobe are also shown in Figure 5. In the reconstructed image the main perturbation is produced close to the correct location with localized changes corresponding to the haematoma. The reconstruction shows the haematoma on the right side and the reconstructed DCP almost follows the DCP of the original object. The center of the reconstructed anomaly on the right side was calculated as (0.4043R, $-0.5282 \mathrm{R})$.

\section{Conclusion}

Two experiments have been considered to investigate the detection of haematoma in sheep's head using our technique. The first experiment, in which simulated blood was injected continuously, does establish that haematoma with a volume of less than $2 \mathrm{ml}$ can be quantified and detected. The exact position of the simulated haematoma could not be detected since the simulated blood was spread out inside the skull in the absence of CSF and brain pressure, and its position was unknown. Therefore the second experiment introduced a new method to simulate haematoma and derive its position. The simulated blood solution was transformed to gel with the same conductivity as blood and placed in a certain position. Simulated haematomas were clearly detected and localised using our developed system. These promising results of sheep experiments prove that the detection and quantification of small haematomas in the brain is possible and encourage further investigation.

\section{References}

[1] T. Mertol, M. Guner, U. Acar, H. Atabay \& U. Kirisoglu, Delayed traumatic intracerebral hematoma, British Journal of Neurosurgery, 5(5), 1991, 491 - 498.

[2] D. YATES, Head Injury: triage, assessment, investigation and early management of head injury in infants, children and adults; Methods, evidence \& guidance (The Royal College of Surgeons of England, London: National Collaborating Centre for Acute Care, 2007).

[3] D. S. Holder, Electrical impedance tomography: Methods, history and applications (GB, Institute of Physics, 2005).

[4] S. Wanjun, Y. Fusheng, Z. Wei, Z. Hongyi, F. Feng, S. Xuetao, L. Ruigang, X. Canhua, D. Xiuzhen, \& B. Tingyi, Image monitoring of an intraperitoneal bleeding model of pigs using electrical impedance tomography, Physiological Measurement, 29(2), 2008, 217-225.

[5] S. Bentolhoda Ayati, K. Bouazza-Marouf, D. Kerr \& M. O'Toole, Performance evaluation of a digital electrical impedance tomography system, In Imaging and Signal Processing in Health Care and Technology (ISPHT 2012), Baltimore, USA, 101-105.

[6] L. A. Geddes \& L. E. Baker, The specific resistance of biological material - a compendium of data for the biomedical engineer and physiologist, Medical \& Biological Engineering, 5(3), 1967, 271-293.

[7] S. Oh, T. Tang, A. Tucker \& R. Sadleir, Normalization of a spatially variant image reconstruction problem in electrical impedance tomography using system blurring properties, Physiological Measurement, 30(3), 2009, 275289.

[8] P. C. Hansen, \& D. P. O'Leary, The use of the L-curve in the regularization of discrete ill-posed problems, SIAM Journal of Science and Computing, 14(6), 1993, 14871503. 